\title{
PHOTOEXCITATION SPECTROSCOPY AND MATERIAL ALTERATION WITH FREE-ELECTRON LASER
}

J. Sturmann, R.G. Albridge, A.V. Barnes, J. Gilligan, M.T. Graham, J.T. Mckinley, A. UedA, W. Wang, X. Yang, N.H. TolK

Center For Molecular and Atomic Studies and Surfaces

Department of Physics and Astronomy, Vanderbilt University

Box 1807-B, Nashville, TN 37235, USA

\section{J.L. DAVIDSON}

Department of Applied and Engineering Sciences, Vanderbilt University

Box 99-B, Nashville, TN 37235, USA

and G. Margaritondo

Institut de Physique Appliquée, École Polytechnique Fédérale 1015 Lausanne, Switzerland

As synchrotron radiation sources have been used for many experiments in the ultraviolet and X-ray regimes, the free-electron laser is an excellent source for a wide array of infrared-photon projects and applications. The free-electron laser delivers a beam of powerful tunable pulsed radiation which provides the opportunity for spatial and temporal localization of the energy delivered at any desired wavelength within the 2-10 $\mu \mathrm{m}$ regime. One application discussed employs the free-electron laser for spectroscopy as a probe of electronic and vibrational structures. Another application uses the free-electron laser beam as a tool for altering materials in a fundamentally new way.

PACS numbers: $63.20 .-\mathrm{e}, 73.20$. At, 79.20.Ds, 79.20.La, 79.60.Jv

\section{The Vanderbilt University free-electron laser}

The Vanderbilt University free-electron laser (VU FEL) delivers infrared beams tunable from $2 \mu \mathrm{m}$ up to $10 \mu \mathrm{m}$. It has demonstrated a sustained average power of $11 \mathrm{~W}$ [1]. The VU FEL macropulse is typically $3-5 \mu$ s long with a repetition rate variable up to $30 \mathrm{~Hz}$ and consists of 1 ps long micropulses separated by $350 \mathrm{ps}$. The typical macropulse energy is $\approx 100-200 \mathrm{~mJ}$. The peak power in a micropulse $\approx 8 \mathrm{MW}$, which may provide up to $\approx 820 \mathrm{GW} / \mathrm{cm}^{2}$ peak irradiation when focused to $\mathrm{a} \approx 50 \mu \mathrm{m}$ spot. Monochromatic (but incoherent) $\mathrm{X}$-rays are also available to users. 


\section{Spectroscopies with the FEL}

\subsection{Internal photoemission}

For many years, concentrated experimental and theoretical research has focused on the band lineup at the interface of two semiconductors [2]. Internal photoemission (IPE) is a simple method of determining the band discontinuities at mature interfaces. We have shown the feasibility of conducting very accurate semiconductor heterojunction band discontinuity measurements using free-electron laser internal photoemission (FELIPE) on AlGaAs/GaAs [3] and GaAs/amorphous-Ge (GaAs/a-Ge) heterojunctions [4].

Unlike conventional photoemission spectroscopies [2] which require the interface to be within a few monolayers of the surface, FELIPE can be used to study interfaces buried deep beneath a surface and the measurements can be made while the interface is under bias.

With FELIPE the lowest IR photon energy, which can induce electronic transitions between the conduction band minima of the two materials, gives directly the conduction band discontinuity. This is much simpler than conventional IPE experiments [5], which use monochromatic visible light inducing transitions from the top of the valence band on one side to the bottom of conduction band on the other side. The knowledge of the interface band gap is required to determine the band discontinuity from visible-IPE data.

The preparation of the GaAs/a-Ge heterojunctions and experiment procedures were described in $[5,6]$. The FEL produces an observable photocurrent by photo-assisting electrons over the conduction band discontinuity as shown in Fig. 1a. A representative spectrum is given in Fig. 1b, showing how $\Delta E_{\mathrm{c}}$ is given directly by the photon energy at the photocurrent threshold.
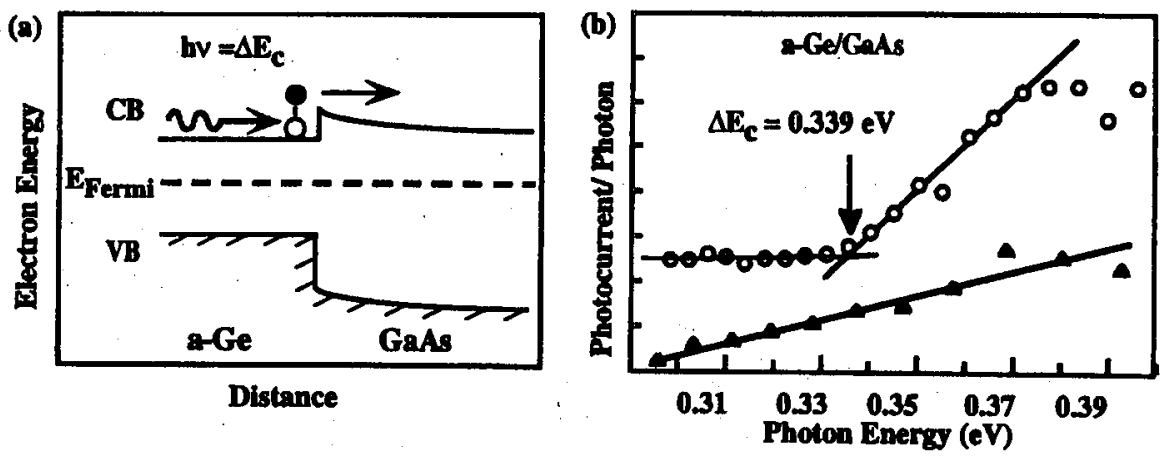

Fig. 1. (a) Energy band diagram of the GaAs/a-Ge interface. If the photon energy exceeds $\Delta E_{\mathrm{c}}$, a photocurrent is produced. (b) Upper lines - linear fit to threshold region for $\mathrm{GaAs} / \mathrm{a}-\mathrm{Ge}$ interface with $-0.8 \mathrm{~V}$ bias. Lower line - photocurrent measured by applying $100 \mathrm{~V}$ between two electrodes attached to the $\mathrm{Ni} / \mathrm{Ga} / \mathrm{Au}$ back-contacts of the $\mathrm{GaAs}$, showing that the photocurrent threshold is not due to bulk GaAs photocurrent. 
A variety of theoretical line shapes have been suggested for the near-threshold region [7], all of the form $Y \propto\left(h \nu-\Delta E_{\mathrm{c}}\right)^{n}$, where $Y$ is the photocurrent yield, $h \nu$ is the photon energy, and $n$ is 2, 2.5 or 3 in the Fowler, Kane and Powell models. When the Fowler fit is replaced by a simple linear fit $(n=1)$, the estimate of $\Delta E_{\mathrm{c}}$ changes by only $5 \mathrm{meV}$. For biases of the a-Ge side relative to the GaAs side of $-0.8 \mathrm{~V}$ and $-1 \mathrm{~V}$, the linear fit gives $\Delta E_{\mathrm{c}}=0.399 \mathrm{eV}$ while the Fowler fit gives $0.334 \mathrm{eV}$. For bias voltages between 0 and $-1 \mathrm{~V}$, no significant variations of $\Delta E_{\mathrm{c}}$ were found. This indicates that tunneling corrections are negligible.

A $100 \mathrm{~V}$ bias was applied across two $\mathrm{Ni} / \mathrm{Ge} / \mathrm{Au}$ contacts on the back of the GaAs. No threshold was observed in the resulting photoconductivity spectrum, therefore the threshold in the spectrum is not a bulk GaAs effect.

As long as flat-band conditions exist at the interface, a direct measurement of the interfacial band gap of the small gap material can be obtained by comparing the results of visible-IPE [8], with those of FELIPE. The near-interface and bulk gaps agreed to within experimental error for the case of GaAs/a-Ge. The first experimental data obtained with this method enabled determination of the discontinuity of a GaAl/AlGaAs heterojunction with high accuracy [4]. This technique has developed to the point that measurements to $5 \mathrm{meV}$ uncertainty are possible.

\subsection{Two-photon absorption}

Two-photon spectroscopy has long been an important component of solid-state research due to its selection-rule complementarity with respect to conventional optical spectroscopy and its demonstration of many interesting and nontrivial features. While certain aspects of this spectroscopy could be practically implemented after the invention of lasers [9], two-photon absorption in germanium had never been thoroughly studied. The FEL provides the technology needed to achieve a positive test of the decades-old Bassani-Hassan predictions.

The results of our Ge two-photon absorption experiments [10] show that the coefficient of indirect two-photon absorption is three orders of magnitude less than the direct gap coefficient. They also demonstrate that indirect absorption is longitudinal optical-phonon (LO-phonon) assisted which provides a positive test of the Bassani-Hassan predictions [11-13].

These results are based on photoconductivity measurements at $\approx 125 \mathrm{~K}$. The sample used for photoconductivity measurements was a $p-i-n$ diode. It was biased at $300 \mathrm{mV}$ in order to fully deplete the intrinsic region before the photoconductivity was measured. Photoconductivity was converted into a voltage change $\Delta V$. Composite data from three different runs was plotted as $\Delta V / V_{\mathrm{r}}^{2}$ vs. twice the photon energy, $2 \hbar \omega$. Fits of the data show that the near-threshold intensity dependence is essentially quadratic. The data in Fig. 2 give a direct gap of $E_{\mathrm{g}}^{\mathrm{d}}=0.87 \pm 0.01 \mathrm{eV}$. Using Mahan's excitonic theory [14], with $E_{\mathrm{g}}^{\mathrm{d}}$ as an adjustable parameter, $0.87 \mathrm{eV}$ was again obtained, confirming the previous evaluation. A gap of $0.87 \mathrm{eV}$ at $125 \mathrm{~K}$ is also consistent with the published value $[15,16]$.

The value of $E_{\mathrm{g}}^{\mathrm{d}}$ obtained from this data analysis was then used to analyze the indirect gap data. It can be safely assumed that the difference between the gaps is independent of temperature, as the published dependence $[15,16]$ is negligible 


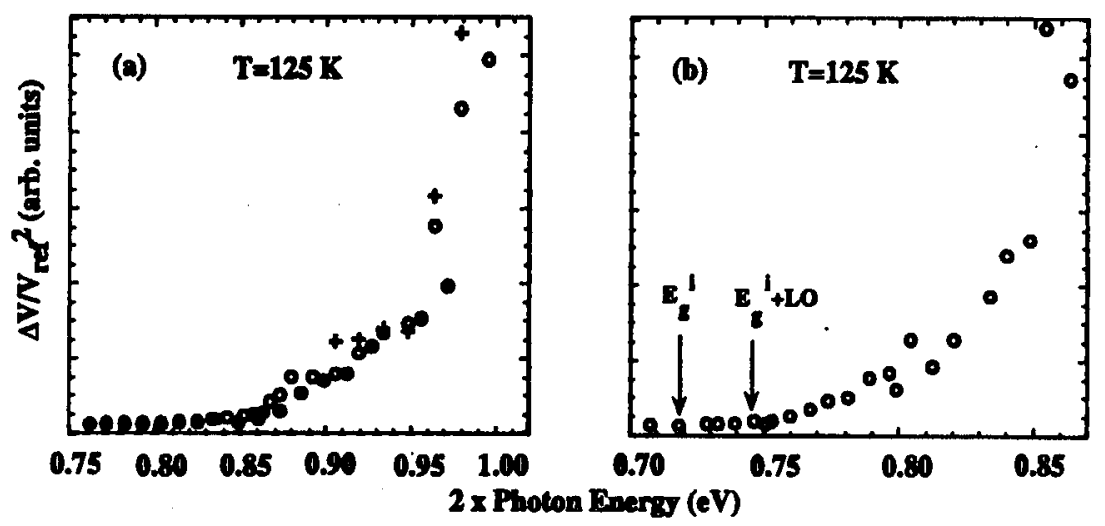

Fig. 2. Two-photon absorption induced photoconductivity signal as a function of double the incident photon energy, with normalization to the reference signal $V_{\text {ref }}$. (a) Threshold indicating a direct gap width of $0.87 \mathrm{eV}$ at $125 \mathrm{~K}$. (b) Similar data for the indirect gap. The pointers mark the predictions of the Bassani-Hassan theory for the indirect gap width $E_{\mathrm{g}}^{\mathrm{i}}$ and the corresponding LO phonon-assisted process.

within the experiment's level of accuracy. From the indirect gap width $[15,16]$ at $77 \mathrm{~K}$ of $0.737 \mathrm{eV}$ and the direct-gap decrease of $19 \mathrm{meV}$ from $77-125 \mathrm{~K}$, an indirect gap width $E_{\mathrm{g}}^{\mathrm{i}}=0.718 \pm 0.01 \mathrm{eV}$ at $125 \mathrm{~K}$ was predicted.

Figure $2 \mathrm{~b}$ shows that the two-photon indirect-gap threshold is clearly shifted with respect to the predicted $E_{\mathrm{g}}^{\mathrm{i}}$. This shift is consistent with a process assisted by the emission of a $30.4 \mathrm{meV}$ LO (L6-) phonon [16], as Bassani and Hassan [11] predicted in 1972. It is conclusive that the two-photon indirect threshold is primarily an LO-assisted process, differing from an one-photon mechanism, which can be assisted by several different types of phonons.

Using our data to estimate the relative strength of the direct and indirect absorption, we found that the indirect absorption was weaker by a factor of $\approx 2 \times 10^{3}$. We reached this approximation by determining the ratio of the absorption at a photon energy of $0.490 \mathrm{eV}$ (above the two-photon direct-gap threshold) to the absorption at a photon energy of $0.407 \mathrm{eV}$ (between the indirect and the direct gaps) and assuming that only two-photon absorption contributes to the photocurrent.

\section{Wavelength-selective materials modification}

\subsection{Laser ablation of diamond}

In recent years, increasing interest has accrued to the clarification of photon induced resonant direct and indirect bond-breaking mechanisms because this method can achieve materials alteration while subjecting the sample to only a fraction of the energy required in a non-localized thermal bond-breaking process.

We have performed wavelength-dependent laser ablation studies on $10 \mu \mathrm{m}$ thick chemical vapor deposition (CVD) diamond films using the VU FEL. Near the 
$3.5 \mu \mathrm{m}$ wavelength, a sharp dip was observed in the massive ablation threshold [17]. This correlates strongly to the $\mathrm{C}-\mathrm{H}$ stretch absorption band, which is present near $3.5 \mu \mathrm{m}$ in the infrared absorption spectra of the CVD diamond samples. The spectra were taken using a Fourier transform infrared (FTIR) spectrometer with $p$-polarized light incident at Brewster's angle. In CVD diamond films, the hydrogen is believed to reside primarily on the surface and at grain boundaries. Consequently, the incoming photon energy is deposited primarily at the surface rather than in the bulk as would be the case for phonon related absorption.

The ablation threshold measurements were conducted in a high vacuum of $10^{-9}$ torr. At each wavelength $(\Delta \lambda \approx 0.05 \mu \mathrm{m})$ a single FEL macropulse was focused $(d \approx 56 \mu \mathrm{m})$ at the sample and the diameter of the resulting hole as a function of laser irradiation was measured using a scanning electron microscope. The massive ablation threshold was defined as an extrapolated value to zero hole diameter.

The initial onset of photodesorption was measured using time-of-flight spectroscopy [18]. A sharp increase in the desorption rate at an intensity of only $3 \mathrm{GW} / \mathrm{cm}^{2}$ was observed at $3.5 \mu \mathrm{m}$. This is markedly different from the graphite, in which case the desorption rate increases relatively gradually starting at an intensity of $0.1 \mathrm{GW} / \mathrm{cm}^{2}$. The $\mathrm{C}-\mathrm{H}$ bond-stretching mode arising from hydrogen on the surface is clearly important in the early stages of the damage process of CVD diamond films.

\subsection{Non-thermal, localized alteration of impurity distributions}

Materials alteration stimulated by the localized absorption of resonant photons has potential applications in the control of selected impurities and formation of clusters. The control of the impurity distribution in a solid requires species-specific deposition of energy exclusively at the impurity site, which is made possible due to the intense IR laser radiation available at the VU FEL.

In these experiments, a sample is exposed to laser light tuned to a special frequency characteristic of a particular impurity. This specific deposition of energy makes it possible to alter the impurity distribution profile and to control cluster configurations. With this technique, the alteration occurs at temperatures and pressures significantly lower than what is typically required for the normal globally thermal activation energy. Diffusion normally involves overcoming the activation energy of an impurity by thermal processes. However, one may locally decrease the activation energy of an impurity by irradiating a sample with a laser tuned to the vibrational frequency of the impurity. An important issue is the degree to which the alteration may be accomplished before the local excitation decays by phonon exchange leading to thermalization. The understanding of this selective chemistry requires a detailed knowledge of the rates and mechanisms of energy transfer from the vibrational modes. The measure of this energy transfer rate is the vibrational lifetime. The longer the lifetime, the more likely it is that we can affect the chemistry. We are developing a technique, applying the pump-probe transient bleaching method [19] to measure vibrational lifetimes using the VU FEL. The IR wavelength range available is ideal to target vibrational modes in various systems. 
Recent measurements have demonstrated that clusters of nitrogen in natural diamond may be dispersed by exposure to FEL radiation tuned to $7.8 \mu \mathrm{m}$ (the vibrations of the clusters) at zero pressure. This effect occurred at temperatures of $600^{\circ} \mathrm{C}$, that is considerably lower than at which nitrogen is normally mobile in diamond. This selective modification is opposite to the thermodynamic tendency for nitrogen to aggregate in diamond. The dispersed nitrogen was identified through electron paramagnetic resonance and by observation of absorption peaks in the FTIR spectrum [20]. Rapid changes in the relative population of the dispersed and aggregate configurations have been demonstrated by other groups, but only under extreme conditions of enormous pressure and high temperature.

The control of the relative populations of nitrogen impurity configurations in diamond is the first experimental demonstration of a new class of technologically-important material processing techniques using selective laser-assisted control of clustering and dispersion of impurities.

Work is underway to demonstrate selective diffusion in silicon and diamond, and to alter different types of cluster species in these same materials. One important goal in this work is to produce much sought after technological grade $n$-type dopant in diamond, a goal which continues to elude researchers. Another possible application will be the control of heterojunction band discontinuities through selective diffusion of impurities near the interface as discussed in the following section.

\subsection{Modification of band offsets}

The band offset of a heterojunction is a significant parameter in device applications. A great deal of effort has been expended to try to tune the magnitude of the offsets to improve electrical properties of devices. So far some success have been achieved.

The offset of a heterojunction is largely determined by the electronic states in the interface. If the states are changed, it is expected that the offset will change. Based on this hypothesis, several groups have fabricated heterojunctions with their offsets altered by nearby impurities [21-23]. The basic idea is that by growing a very thin intralayer close to the interface, an extra dipole is formed near the heterojunction. To minimize this dipole, the energy band has to adjust which results in an altered band offset value. These groups were successful, however the alteration was carried out during the growth of the heterojunction. After growth, the band offset cannot be further altered with the above method.

We proposed a new way to change the band offset of heterojunctions after its growth with the use of the FEL. Defects and impurities at the interface determine the interface states. Therefore, any change in the defect and impurity distribution at the interface may cause significant changes in the electron states at the inter- face which in turn will change the band offset. The photon energy of the FEL lies within the range of the vibrational energies of many of these impurities and defects: We can tune the wavelength of the FEL to those vibrational energies and can selectively cause the migration of the desired impurities and/or defects. This non-thermal migration will change the electronic states at the interface which will consequently adjust the band offsets at the interface. 


\section{Acknowledgments}

This work was partially supported by the Office of Naval Research under contracts No. N00014-87-C-0146, No. N00014-91-C-109. The authors gratefully acknowledge the staff of the Vanderbilt Free-Electron Laser Center.

\section{References}

[1] C.A. Brau, Nucl. Instrum. Methods Phys. Res. A 318, 38 (1992).

[2] F. Capasso, G. Margaritondo, Heterojunction Band Discontinuities: Physics and Device Applications, North-Holland, Amsterdam 1987.

[3] C. Coluzza, E. Tuncel, J.L. Staheli, P.A. Baudat, G. Margaritondo, J.T. McKinley, A. Ueda, A.V. Barnes, R.G. Albridge, N.H. Tolk, D. Martin, F. Morier-Genoud, C. Dupuy, A. Rudra, M. Ilegems, Phys. Rev. B 46, 12834 (1992).

[4] J.T. McKinley, R.G. Albridge, A.V. Barnes, A. Ueda, N.H. Tolk, C. Coluzza, F. Gozzo, G. Margaritondo, D. Martin, F. Morier-Genoud, C. Dupuy, A. Rudra, M. Ilegems, J. Vac. Sci. Technol. B 11, 1614 (1993).

[5] C. Coluzza, A. Neglia, A. Bennouna, M. Capizzi, R. Carluccio, A. Frova, P.C. Srivastava, Appl. Surf. Sci. 56-58, 733 (1992).

[6] C. Coluzza, F. Lama, A. Frova, P. Perfetti, C. Quaresima, M. Capozi, J. Appl. Phys. 64, 3304 (1988).

[7] R.H. Fowler, Phys. Rev. 38, 45 (1931); E.O. Kạne, Phys. Rev. 147, 335 (1966); R.J. Powell, J. Appl. Phys. 41, 2424 (1970).

[8] G. Abstreiter, U. Prechtel, G. Weismann, W. Schlapp, Physica B 134, 433 (1985); G. Abstreiter, U.U. Prechtel, G. Weismann, W. Schlapp, Surf. Sci. 174, 312 (1986).

[9] V. Nathan, A.H. Guenther, S.S. Mitra, J. Opt. Soc. Am. B 2, 294 (1985).

[10] E. Tu ncel, J.L. Staheli, C. Coluzza, G. Margaritondo, J.T. McKinley, R.G. Albridge, A.V. Barnes, A. Ueda, X. Yang, N.H. Tolk, Phys. Rev. Lett. 70, 4146 (1993).

[11] F. Bassani, A.R. Hassan, Nuovo Cimento B 7, 313 (1972).

[12] F. Bassani, G. Pastori-Parravicini, Electronic States and. Optical Transitions in Solids. Pergamon, Oxford 1975.

[13] A.R. Hassan, Nuovo Cimento B 13, 19 (1973).

[14] G.D. Mahan, Phys. Rev. 170, 825 (1968).

[15] T.P. McLean, in: Progress in Semiconductors, Vol. 5, Ed. A.F. Gibsen, Heywood, London 1960, p. 53.

[16] Landolt-Börnstein, Numerical Data and Functional Relationships in Science and Technology, Gp. III, Vol. 17a, Eds. M. Cardona, G. Harbeke, A. Rössler, P. Madelung, Springer, Berlin 1982, p. 87.

[17] A. Ueda, R.G. Albridge, A.V. Barnes, A. Burger, W.E. Collins, J.L. Davidson, M.A. George, Z. Hu, M.L. Languell, J.T. McKinley, J. Tribble, N.H. Tolk, Nucl. Instrum. Methods Phys. Res. B 100, 427 (1995).

[18] A. Ueda, J.T. McKinley, R.G. Albridge, A.V. Barnes, N.H. Tolk, J.L. Davidson, M.L. Languell, Mater. Res. Soc. Symp. Proc. 285, 215 (1992).

[19] E.J. Heilweil, M.P. Casassa, R.R. Cavanagh, J.C. Stephenson, J. Chem. Phys. 82, 5216 (1985).

[20] M.T. Graham, J.T. McKinley, A. Beth, R.G. Albridge, A.V. Barnes, J.L. Davidson, N.H. Tolk, to be published in Proc. 13th Int. Conf. on Defects in Insulating Materials, Wake Forest U., Winston-Salem, NC, 1996. 
[21] F. Capasso, A.Y. Cho, K. Mohammed, P.W. Foy, Appl. Phys. Lett. 46, 664 (1985).

[22] G. Muller, A. Zrenner, F. Koch, K. Ploog, Appl. Phys. Lett. 55, 1564 (1989).

[23] T.H. Shen, M. Elliot, R.H. Williams, D. Westwood, Appl. Phys. Lett. 58, 842 (1991). 